\title{
Tratamento Quimioterápico do Mesotelioma Pleural Maligno: Revisão Sistemática
}

https://doi.org/10.32635/2176-9745.RBC.2019v65n1.348

\author{
Malignant Pleural Mesothelioma Chemotherapy Treatment: Systematic Review \\ Tratamiento Quimioterápico del Mesotelioma Pleural Maligno: Revisión Sistemática
}

\author{
André de Oliveira Souza'; Vera Lúcia Edais Pepe²; Lenice Gnocchi da Costa Reis³; Rosângela Caetano4; Luiz Gustavo André Oliveira ${ }^{5}$
}

Resumo

Introduçáo: O mesotelioma pleural maligno é um câncer raro, agressivo e que apresenta expectativa de aumento na incidência até 2030. As melhores formas de tratar essa neoplasia continuam em debate. Objetivo: Sintetizar as evidências de eficácia e segurança dos esquemas quimioterápicos de primeira linha disponíveis para o tratamento do mesotelioma pleural maligno. Método: Foram utilizadas as bases bibliográficas LILACS, MEDLINE, Scopus, Cochrane Controlled Trials Register e Web of Science. Buscaram-se estudos na literatura cinzenta. Os critérios de elegibilidade incluíram ensaios randomizados de fases II ou III, de pacientes com mesotelioma pleural virgem de tratamento quimioterápico, submetidos a qualquer regime terapêutico, tendo como controle outros esquemas quimioterápicos ou controle ativo de sintomas, e apresentando tempo de sobrevida global, tempo livre de progressão, resposta tumoral e toxicidade como desfechos. Todas as etapas foram realizadas por dois revisores, de forma independente. O protocolo da revisão foi registrado no International Prospective Register of Systematic Reviews (PROSPERO 2014: CRD42014014388). Resultados: Treze estudos envolvendo 14 esquemas terapêuticos foram incluídos. O único esquema quimioterápico que se apresentou superior ao comparado com significância estatística nos três desfechos de eficácia foi cisplatina + pemetrexede. Cisplatina + pemetrexede e cisplatina + gemcitabina apresentaram mais casos de toxicidade graus 3 e 4. Conclusão: Existem boas evidências para recomendar combinaçóes de derivado de platina e antifolato como opção de primeira escolha no tratamento quimioterápico do mesotelioma pleural. Mais estudos clínicos são necessários para embasar decisóes de incorporaçáo dos antifolatos no tratamento rotineiro dessa neoplasia no Brasil.

Palavras-chave: Mesotelioma; Tratamento Farmacológico; Neoplasias Pulmonares.

\begin{abstract}
Introduction: Malignant pleural mesothelioma is a rare, aggressive cancer that is expected to increase in incidence by 2030 . The best ways to treat this neoplasm are still under discussion. Objective: To synthesize the evidence of efficacy and safety of the different first-line chemotherapy regimens available for the treatment of malignant pleural mesothelioma. Method: The LILACS, MEDLINE, Scopus, Cochrane Controlled Trials Register and Web of Science bibliographic databases were used. Studies were sought in the grey literature. Eligibility criteria included randomized phase II or III trials of chemotherapy-naive patients with pleural mesothelioma who underwent any therapeutic regimen, compared to other chemotherapeutic regimens or active symptom control, and presenting overall survival, progression free survival, tumor response and toxicity as outcomes. All steps were performed independently by two reviewers. The review protocol was recorded in the International Prospective Register of Systematic Reviews (PROSPERO 2014: CRD42014014388). Results: Thirteen studies involving fourteen therapeutic regimens were included. The only chemotherapy regimen that presented superior to the comparator with statistical significance in the three efficacy outcomes was cisplatin + pemetrexed. Cisplatin + pemetrexed and cisplatin + gemcitabine presented more grades 3 and 4 toxicity cases. Conclusion: There is good evidence to recommend combinations of platinum and antifolate derivatives as a first-choice option in the chemotherapeutic treatment of pleural mesothelioma. Further clinical studies are needed to support decisions to incorporate antifolates in the routine treatment of this neoplasm in Brazil.

Key words: Mesothelioma; Drug Therapy; Lung Neoplasms.
\end{abstract}

Resumen

Introducción: El mesotelioma pleural maligno es un cáncer raro, agresivo y que presenta expectativa de aumento en la incidencia hasta 2030. Objetivo: Sintetizar las evidencias de eficacia y seguridad de los diferentes esquemas quimioterápicos de primera línea disponibles para el tratamiento del mesotelioma pleural maligno. Método: Se utilizaron las bases bibliográficas LILACS, MEDLINE, Scopus, Cochrane Controlled Trials Register y Web of Science. Se buscó estudios en la literatura gris. Los criterios de elegibilidad incluyeron ensayos aleatorizados de fase II o III, de pacientes con mesotelioma pleural vírgenes de tratamiento quimioterápico, sometidos a cualquier régimen terapéutico, teniendo como control otros esquemas quimioterápicos o control activo de síntomas, y presentando tiempo de supervivencia global, tiempo libre de progresión, respuesta tumoral y toxicidad como resultados. Todas las etapas fueron realizadas por dos revisores, de forma independiente. El protocolo de la revisión se registró en el International Prospective Register of Systematic Reviews (PROSPERO 2014: CRD42014014388). Resultados: Se incluyeron trece estudios de catorce esquemas terapéuticos. El único esquema quimioterápico que se presentó superior al comparador con significancia estadística en los tres resultados de eficacia fue cisplatino + pemetrexede. Cisplatino + pemetrexed y cisplatino + gemcitabina presentaron más casos de toxicidad grados $3 \mathrm{y}$ 4. Conclusion: Existen buenas evidencias para recomendar combinaciones de derivado de platino y antifolato como opción de primera elección en el tratamiento quimioterápico del mesotelioma pleural. Más estudios clínicos son necesarios para basar decisiones de incorporación de los antifolatos en el tratamiento rutinario de esa neoplasia en Brasil.

Palabras clave: Mesotelioma; Tratamiento Farmacológico; Neoplasias Pulmonares.

\footnotetext{
${ }^{1}$ Instituto Nacional de Câncer José Alencar Gomes da Silva (INCA). Rio de Janeiro (RJ), Brasil. Orcid iD: https://orcid.org/0000-0002-7639-606X

${ }^{2}$ Escola Nacional de Saúde Pública Sérgio Arouca (Ensp). Rio de Janeiro (RJ), Brasil. Orcid iD: https://orcid.org/0000-0003-0606-1544

${ }^{3}$ Ensp. Rio de Janeiro (RJ), Brasil. Orcid iD: https://orcid.org/0000-0001-5020-2469

${ }^{4}$ Ensp. Rio de Janeiro (RJ), Brasil. Orcid iD: https://orcid.org/0000-0003-1480-2453

${ }^{5}$ Fundação do Câncer. Rio de Janeiro (RJ), Brasil. Orcid iD: https://orcid.org/0000-0002-5895-1170

Endereço para correspondência: André de Oliveira Souza. Rua Cosmorama, 193, apto. 7, bloco B - Cosmorama. Mesquita (RJ), Brasil. CEP 26582-020. E-mail: andreindio@yahoo.com.br
} 


\section{INTRODUÇÃO}

O mesotelioma pleural maligno é um câncer ocupacional originado nas células mesoteliais e submesoteliais da pleura. É uma neoplasia rara, agressiva e, por vezes, difícil de diferenciar do adenocarcinoma de pulmão. Seus principais sintomas são dor torácica, dispneia e perda de peso, que acontecem de forma progressiva, usualmente de diagnóstico tardio e em fases já avançadas da doença ${ }^{1}$. Independentemente do tipo histológico, a sobrevida após o diagnóstico é pequena ${ }^{2}$.

Mais de $80 \%$ dos casos de mesotelioma pleural maligno estão relacionados à exposição profissional prévia ao asbesto e existe uma relação dose-resposta com as áreas de incidência mais elevadas nos locais com indústrias que utilizam intensamente o mineral ${ }^{3,4}$. Apesar da proibição do asbesto em diversos países e Estados brasileiros, a incidência do mesotelioma pleural maligno é crescente. Em razão do longo período de latência desses tumores, não é esperada sua queda até $2030^{5}$.

O Brasil é o maior produtor, consumidor e exportador mundial de amianto e, aproximadamente, $300 \mathrm{mil}$ trabalhadores estavam expostos ao produto em $2010^{6}$. Estudos sobre câncer relacionados à exposiçáo ao amianto são ainda escassos no país, em virtude principalmente das dificuldades diagnósticas e sub-registro dos casos. Foram registrados 2.308 óbitos por mesoteliomas entre 2000 a 2012 no país; mas, estima-se que esse número esteja subestimado, sendo esperado que a mortalidade por esse câncer continue a crescer no país pelos próximos 15 a 20 anos $^{6}$.

A abordagem diagnóstica e terapêutica dessa neoplasia permanece difícil e complexa ${ }^{2}$. Seu tratamento é multimodal, composto por cirurgia, radioterapia e quimioterapia. Pacientes candidatos ao tratamento com finalidade curativa possuem sobrevida média de 13 a 47 meses e, nos submetidos a tratamento paliativo, raramente ultrapassa oito a 12 meses. Tipo histológico não epitelial, alto performance status, estágios avançados da doença, idade acima de 49 anos, gênero masculino, dor torácica, perda de peso, baixo nível de hemoglobina, trombocitose, leucocitose e alto nível de lactato desidrogenase estáo associados a pior prognóstico ${ }^{2}$.

A maioria dos pacientes possui doença irressecável ao diagnóstico ou é inoperável em razão da idade ou presença de comorbidades clínicas, sendo usualmente tratados com quimioterapia sistêmica, objetivando prolongar a sobrevida e melhorar a qualidade de vida ${ }^{7}$. Existem controvérsias sobre os melhores esquemas terapêuticos para a doença, com alguns casos tornando-se eventualmente resistentes.

Não existe ainda no país um protocolo clínico ou diretriz terapêutica sobre a doença, e a sua raridade dificulta a obtenção de amostras com o poder estatístico necessário para a realização de ensaios clínicos de alta qualidade. Além disso, a expectativa de que o número de mesoteliomas pelo uso do amianto venha a aumentar com o passar do tempo faz com que seja importante disponibilizar a informação existente sobre a eficácia e segurança dos esquemas terapêuticos utilizados no tratamento medicamentoso do mesotelioma pleural maligno.

Esta revisão sistemática sintetizou as evidências acerca da eficácia e da segurança dos esquemas quimioterápicos de primeira linha disponíveis para o tratamento do mesotelioma pleural maligno.

\section{MÉTODO}

O protocolo da revisão foi registrado no International Prospective Register of Systematic Reviews (PROSPERO 2014:CRD42014014388). A revisão considerou apenas ensaios clínicos randomizados de tratamento quimioterápico em pacientes com mesotelioma pleural maligno virgens de tratamento, de fases II ou III.

Foram utilizadas as bases bibliográficas MEDLINE (via Pubmed), LILACS, Scopus, Cochrane Controlled Trials Register e Web of Science. Também foi consultada a base de ensaios clínicos Clinical Trials. As buscas foram realizadas em março de 2019, sem delimitaçáo de periodo inicial para recuperação das referências.

Estratégias de busca, adaptadas à base pesquisada e delineadas com auxílio de um bibliotecário, incluíram a pesquisa de descritores ou de palavras no texto relacionados à doença ["mesothelioma", "pleural neoplasms", "pleural cancer"], tipo de intervenção ["drug therapy", "antineoplastic agents", "chemothera-py"] e tipo de estudo ["randomized controlled trial", "controlled clinical trial", "random allocation]. A estratégia completa de pesquisa pode ser obtida por contato com os autores.

A estratégia de busca para a base Medline (via Pubmed) foi a seguinte: (((eothelioma[mh] OR Mesothelioma[ti]) AND (Pleural Neoplasms[mh] OR Pleural[ti]) AND (drug therapy[sh] OR drug therapy[tiab] OR drug*[tiab] OR drug therapy[tw] OR "antineoplastic agents"[All Fields] OR "antineoplastic agents" [mh] OR antineoplastic agents[tw] OR chemotherap*[tiab])) AND (randomized controlled trial[pt] OR controlled clinical trial[pt] OR randomized controlled trials[mh] OR random allocation $[\mathrm{mh}]$ OR double-blind method[mh] OR single-blind method [mh] OR clinical trial[pt] OR clinical trials[mh] OR ("clinical trial”" $[\mathrm{tw}])$ OR ((singl* $[\mathrm{tw}]$ OR

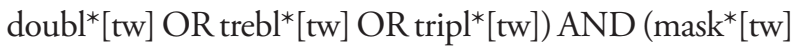
OR blind*[tw])) OR (placebos[mh] OR placebo*[tw] OR random*[tw] OR research design[mh:noexp] OR 
comparative study[mh] OR evaluation studies[mh] OR follow-up studies[mh] OR prospective studies[mh] OR control* $^{*}[\mathrm{tw}]$ OR prospectiv* $[\mathrm{tw}]$ OR volunteer* $\left.\left.{ }^{*} \mathrm{tw}\right]\right)$ NOT (animals[mh] NOT humans[mh]))).

Foram examinadas as referências bibliográficas dos estudos clínicos incluídos nessa revisão sistemática, nas revisōes e diretrizes clínicas citadas neste trabalho e em Anais de Congressos recuperados nas bases bibliográficas pesquisadas, na busca de artigos não identificados.

Foram critérios de inclusão: (a) ensaios clínicos randomizados contendo grupos de comparação, nos quais a quimioterapia fosse utilizada como tratamento exclusivo ou fizesse parte do tratamento mutimodal (em combinação com cirurgia ou radioterapia); (b) estudos utilizando como grupo controle esquema quimioterápico ou placebo; (c) ensaios apresentando como desfechos tempo de sobrevida global, tempo livre de progressão, resposta tumoral e toxicidade (eventos adversos); (d) trabalhos publicados nos idiomas inglês, português, espanhol e francês. Estudos contendo participantes com outras neoplasias ou outros tipos de mesoteliomas, quando permitiam a extração em separado dos dados dos participantes com mesotelioma pleural maligno, foram também incluídos. Excluíram-se estudos clínicos sem grupo controle comparativos ou examinando tratamento quimioterápico de segunda linha; editoriais, cartas ou revisóes.

As citaçôes foram armazenadas e manuseadas no software gerenciador de referências (EndNote). Duplicatas de referências foram eliminadas. As referências correspondentes a Anais de Congressos Científicos tiveram suas referências bibliográficas examinadas em busca de estudos que contemplassem os critérios de elegibilidade.

A seleção de estudos foi realizada por dois revisores e as divergências foram resolvidas por consenso. Foram duas etapas: análise dos títulos e os resumos e leitura dos textos completos. A ficha de extraçáo foi elaborada com as seguintes informaçóes: a) informaçóes dos autores: ano de publicação, intervenção, comparador e desfechos reportados; b) características do estudo: número de participantes, tipo histológico, estadiamento da doença, extensão e acesso ao tumor, número de centros de pesquisa e países envolvidos, tempo médio de diagnóstico, tempo médio de seguimento e as perdas de seguimento; c) características dos participantes: idade, sexo, raça, performance status e tempo de duraçáo dos sintomas; d) sobre o tratamento medicamentoso: finalidade da quimioterapia, tratamento multimodal, tratamento anterior e terapia concomitante; e) sobre os desfechos: as definiçóes de cada desfecho segundo os autores dos estudos foram extraídas e comparadas, além dos resultados para os desfechos de interesse e a toxicidade dos esquemas terapêuticos.
Foram examinados os seguintes desfechos: tempo de sobrevida global, definido como o tempo decorrido da entrada do participante no estudo até a morte; tempo livre de progressão da doença, correspondente ao intervalo entre a entrada do participante no estudo até a progressão da doença ou morte; resposta tumoral, referente à taxa de participantes que apresentaram alguma resposta ao tratamento, sendo esta medida de duas maneiras: taxa de resposta objetiva (TRO), relativa à taxa de participantes que apresentaram resposta total ou parcial ao tratamento, e taxa de controle da doença (TCD), representada pelo somatório da TRO e dos participantes que tiveram a doença estabilizada. A toxicidade foi representada pela ocorrência de eventos adversos associados aos diferentes esquemas quimioterápicos, sendo considerada a presença de toxicidade de qualquer grau e, especificamente, dos graus 3 (grave), 4 (com risco de óbito ou incapacidadeo) e 5 (óbito associado ao evento adverso).

A avaliação da qualidade metodológica dos estudos disponíveis em texto completo levou em consideraçáo o processo de randomização, sigilo de alocaçáo, cegamento, análise por intenção de tratar e perdas durante os estudos, fatores considerados mais relevantes pela Cochrane na avaliação de risco de vieses em ensaios clínicos controlados randomizados ${ }^{8}$. Também foram utilizados dois revisores independentes e resolução das divergências por consenso.

Os resultados médios dos desfechos e respectivos intervalos de confiança de $95 \%$ dos esquemas terapêuticos foram registrados como se apresentavam nos manuscritos. Foi construído gráfico forest plot para o tempo de sobrevida global no software Stata versão 12.0, utilizando as diferenças médias padronizadas para expressar o efeito dos tratamentos, com intervalos de confiança de $95 \%$. A diferença média padronizada foi obtida pela razão da diferença média dos resultados do desfecho entre os grupos e o desvio-padrão desses resultados entre os participantes do estudo. Quando não havia dados suficientes para calcular o desvio-padrão das médias do desfecho, adotou-se a média dos desvios-padróes dos outros estudos presentes na mesma análise ${ }^{8}$. Dados de eventos adversos foram relatados como número absoluto e frequência relativa, tais como presentes nos ensaios clínicos.

A presença de grande heterogeneidade dos resultados dos estudos, decorrentes da presença de diferentes esquemas terapêuticos e grupos controles, impediu a realização de metanálises.

\section{RESULTADOS}

Foram identificados 4.136 estudos nas bases pesquisadas e 32 na busca por referência cruzada e em outras fontes. Após a eliminação de 1.144 duplicatas, os títulos e resumos 
restantes foram examinados, sendo excluídos 2.996. Dos 28 trabalhos avaliados como texto completo, 13 estudos preencheram os critérios de elegibilidade e foram incluídos na revisão ${ }^{9-21}$. Dez estavam publicados como artigos completos $^{9-16,19,21}$ e três como resumos de congresso ${ }^{17,18,20}$. A Figura 1 sumariza os resultados das etapas de seleção e os motivos de exclusão.

A Tabela 1 apresenta as principais características referentes aos estudos e aos participantes. O número de participantes entre os trabalhos variou de 25 a 448 (mediana 87), com apenas quatro ensaios incluindo mais de 100 pacientes. As medianas de idade variaram de 56 a 72 anos. Nos 11 estudos que traziam informaçáo sobre tipo histológico do mesotelioma pleural maligno, predominou o tipo epitelial ${ }^{9-13,15,16,18-21}$. A proporção de pacientes com performance status 0-1 foi superior a $80 \%$ em oito estudos ${ }^{9-12,15-17,19}$. Em 11 estudos, apenas participantes sem indicação cirúrgica foram elegíveis ${ }^{9-15,17-20}$. Nos sete estudos que informavam tempo de seguimento, este variou de 10 a 45 meses, $11-13,15,16,19$. Os estudos envolveram 14 esquemas terapêuticos diferentes, sendo os mais frequentes os regimes que combinavam cisplatina associada ao pemetrexede e carboplatina associada ao pemetrexede, com ou sem a presença de outro antineoplásico.

A avaliação da qualidade metodológica dos artigos completos pode ser vista na Figura 2. A qualidade desses trabalhos foi, de modo geral, ruim. Apenas cinco estudos detalharam o processo de randomizaçãa ${ }^{11-13,15,16}$ e só dois referiam o sigilo na alocação dos participantes ${ }^{11,16}$. Apenas um estudo foi realizado como duplo-cego ${ }^{21}$. O fluxo dos participantes e as perdas foram descritos de forma clara em oito trabalhos ${ }^{10-13,15,16,19,21}$. Os mesmos oito estudos foram os que analisaram os resultados por intenção de tratar.

Os esquemas terapêuticos e os resultados dos desfechos examinados estão apresentados na Tabela 2. Os esquemas quimioterápicos que se destacaram com taxas de resposta objetiva acima de $30 \%$ foram carboplatina + pemetrexede; cisplatina + gemcitabina; cisplatina + pemetrexede + CBP501; cisplatina + pemetrexede + axitinib, cisplatina + pemetrexede + bevacizumabe, cisplatina + pemetrexede + nintedanib e cisplatina + pemetrexede + cediranib. Combinação de carboplatina e pemetrexede atingiu resposta objetiva de quase $80 \%$.

A TCD correspondente ao somatório de participantes com resposta objetiva e dos que tiveram a doença estabilizada pela quimioterapia foi superior a $80 \%$ para os esquemas terapêuticos cisplatina associada à gemcitabina e carboplatina associada ao pemetrexede.

Os esquemas terapêuticos vinorelbina e aqueles que associavam o uso de cisplatina com gemcitabina, de cisplatina com pemetrexede; de cisplatina, pemetrexede e axitinib; de cisplatina, pemetrexede e bevacizumabe; cisplatina, pemetrexede e nintedanib; cisplatina, pemetrexede e cediranib; e derivado de platina, pemetrexede
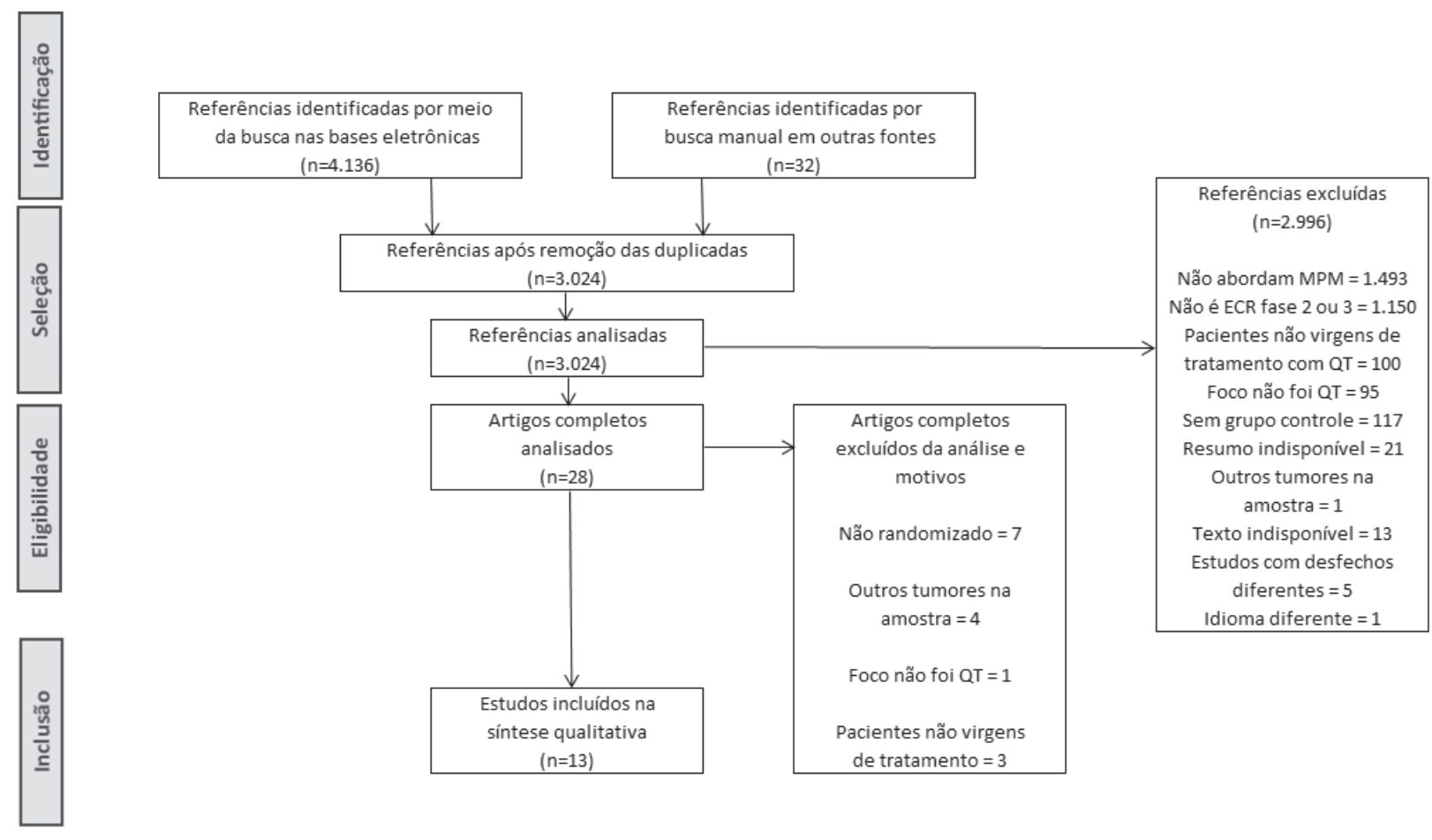

Figura 1. Fluxograma das etapas de seleção de estudos sobre o tratamento quimioterápico do mesotelioma pleural maligno

Legendas: ECR - Ensaio Clínico Randomizado; MPM - Mesotelioma Pleural Maligno, QT - Quimioterapia. 
Tabela 1. Caraterísticas dos estudos e participantes incluídos na revisão sistemática

\begin{tabular}{|c|c|c|c|c|c|c|}
\hline Autor, ano & $\begin{array}{c}\text { Período } \\
\text { do estudo }\end{array}$ & $\begin{array}{c}\text { Tempo de } \\
\text { seguimento } \\
\text { (meses) }\end{array}$ & $\begin{array}{c}\text { Número } \\
\text { de } \\
\text { pacientes }\end{array}$ & $\begin{array}{l}\text { Mediana de } \\
\text { idade em } \\
\text { anos (faixa) }\end{array}$ & Intervenção & Controle \\
\hline $\begin{array}{l}\text { White et al., } \\
2000\end{array}$ & 1994-1997 & - & 25 & $56(28-72)$ & $\begin{array}{c}\text { Cisplatina + } \\
\text { etoposídio }\end{array}$ & Carboplatina \\
\hline $\begin{array}{l}\text { Vogelzang et } \\
\text { al.,2003 }\end{array}$ & $1999-2001$ & 10,0 & 448 & $61(19-85)$ & $\begin{array}{l}\text { Cisplatina }+ \\
\text { pemetrexede }\end{array}$ & Cisplatina \\
\hline $\begin{array}{l}\text { van } \\
\text { Meerbeeck et } \\
\text { al.,2005 }\end{array}$ & $2000-2004$ & 24 & 250 & $58(19-80)$ & $\begin{array}{l}\text { Cisplatina + } \\
\text { raltitrexede }\end{array}$ & Cisplatina \\
\hline $\begin{array}{l}\text { Muers et } \\
\text { al.,2008 }\end{array}$ & 2001-2006 & 36,4 & 409 & $65(46-85)$ & $\begin{array}{l}\text { Cuidados de } \\
\text { suporte + } \\
\text { cisplatina + } \\
\text { mitomicina } \\
\text { + vinblastina } \\
\text { ou cuidados } \\
\text { de suporte + } \\
\text { vinorelbina† }\end{array}$ & $\begin{array}{l}\text { Cuidados de } \\
\text { suporte }\end{array}$ \\
\hline $\begin{array}{l}\text { Millenson et } \\
\text { al., } 2010\end{array}$ & 2005-2007 & - & 32 & $71(53-80)$ & $\begin{array}{c}\text { Gemcitabina }+ \\
\text { pemetrexede }\end{array}$ & $\begin{array}{c}\text { Carboplatina }+ \\
\text { pemetrexede }\end{array}$ \\
\hline $\begin{array}{l}\text { Habib et al., } \\
2013\end{array}$ & 2008-2011 & $18(6-30)$ & 40 & $57(28-74)$ & $\begin{array}{l}\text { Cisplatina + } \\
\text { gemcitabina }\end{array}$ & $\begin{array}{c}\text { Carboplatina }+ \\
\text { pemetrexede }\end{array}$ \\
\hline $\begin{array}{l}\text { Krug et al., } \\
2014\end{array}$ & 2008-2011 & - & 65 & $65(35-84)$ & $\begin{array}{c}\text { Cisplatina }+ \\
\text { pemetrexede }+ \\
\text { CBP501 }\end{array}$ & $\begin{array}{l}\text { Cisplatina + } \\
\text { pemetrexede }\end{array}$ \\
\hline $\begin{array}{l}\text { Zaleman et } \\
\text { al., } 2016\end{array}$ & $2008-2014$ & $\begin{array}{c}39,4 \\
(25,5-54,8)\end{array}$ & 448 & $\begin{array}{c}65,7 \\
(61,3-70,2)\end{array}$ & $\begin{array}{c}\text { Cisplatina + } \\
\text { pemetrexede + } \\
\text { bevacizumabe }\end{array}$ & $\begin{array}{l}\text { Cisplatina + } \\
\text { pemetrexede }\end{array}$ \\
\hline $\begin{array}{l}\text { Buikhuisen et } \\
\text { al., } 2016\end{array}$ & 2009-2012 & 45 & 32 & $61(35-75)$ & $\begin{array}{l}\text { Cisplatina }+ \\
\text { pemetrexede } \\
\text { +axitinib }\end{array}$ & $\begin{array}{l}\text { Cisplatina + } \\
\text { pemetrexede }\end{array}$ \\
\hline $\begin{array}{l}\text { Tsao et al., } \\
2018\end{array}$ & $2011-2018$ & - & 92 & 72 & $\begin{array}{c}\text { Cisplatina }+ \\
\text { pemetrexede }+ \\
\text { cediranib }\end{array}$ & $\begin{array}{l}\text { Cisplatina + } \\
\text { pemetrexede }\end{array}$ \\
\hline $\begin{array}{l}\text { Zauderer et } \\
\text { al., } 2017\end{array}$ & $2011-2015$ & - & 46 & $68(34-84)$ & $\begin{array}{c}\text { Derivado de } \\
\text { platina }+ \\
\text { pemetrexede }+ \\
\text { galinpepimut-S }\end{array}$ & $\begin{array}{l}\text { Derivado de } \\
\text { platina }+ \\
\text { pemetrexede }\end{array}$ \\
\hline $\begin{array}{l}\text { Grosso et al., } \\
2017\end{array}$ & 2013-2017 & $\begin{array}{c}29,0 \\
(26,9-33,1)\end{array}$ & 87 & $67(39-80)$ & $\begin{array}{c}\text { Cisplatina + } \\
\text { pemetrexede }+ \\
\text { nintedanib }\end{array}$ & $\begin{array}{l}\text { Cisplatina + } \\
\text { pemetrexede }\end{array}$ \\
\hline $\begin{array}{l}\text { Kovac et al., } \\
2017\end{array}$ & $2017^{*}$ & - & 96 & 63 & $\begin{array}{l}\text { Cisplatina + } \\
\text { gemcitabina }\end{array}$ & $\begin{array}{l}\text { Cisplatina + } \\
\text { pemetrexede }\end{array}$ \\
\hline
\end{tabular}

Notas: $\dagger$ - No estudo de Muers ${ }^{11}, 136$ pacientes foram submetidos a tratamento com cuidado de suporte + vinorelbina e 137 a cuidados de suporte + mitomicina + vimblastina + cisplatina. ${ }^{*} \mathrm{O}$ estudo de Kovac et al. apresenta somente o ano do seu término.

Processo de randomização Sigilo de alocação

Mascaramento

Análise por intenção de tratar

Perdas durante o estudo

\begin{tabular}{|c|c|c|c|c|c|c|c|c|c|}
\hline Habib & Krug & Muers & Grosso & Zaudere & White & Buikhuisen & Zalcman & Van Meerbeeck & k Vogelzang \\
\hline (?) & (?) & $\oplus$ & $\Theta$ & $\odot$ & (?) & $\oplus$ & $\oplus$ & 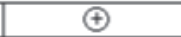 & (†) \\
\hline$\Theta$ & $\Theta$ & 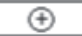 & $\odot$ & $\Theta$ & $\Theta$ & $\Theta$ & $\oplus$ & $\Theta$ & $\odot$ \\
\hline$\odot$ & $\odot$ & $\odot$ & (?) & $\oplus$ & $\odot$ & $\odot$ & $\odot$ & $\odot$ & $\Theta$ \\
\hline$\Theta$ & $(†$ & $\oplus$ & (†) & $\oplus$ & $\Theta$ & $\oplus$ & $\oplus$ & $\oplus$ & 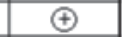 \\
\hline$\odot$ & $\oplus$ & $\oplus$ & $\oplus$ & (†) & (?) & (†) & $\oplus$ & $\oplus$ & $\oplus$ \\
\hline
\end{tabular}

Figura 2. Síntese da avaliação da qualidade dos estudos incluídos na revisão sistemática

Legendas: +: Realizado(a); - : Não realizado(a); ? : Não está claro. 
Tabela 2. Medidas de desfechos dos estudos incluídos, segundo esquemas quimioterápicos para tratamento do mesotelioma maligno de pleura

\begin{tabular}{|c|c|c|c|c|c|}
\hline Esquema & Autor, ano & TRO \% (IC 95\%) & TCD \% (IC 95\%) & $\begin{array}{c}\text { TLP (meses) (IC } \\
95 \%)\end{array}$ & $\begin{array}{c}\text { TSG (meses) (IC } \\
95 \%)\end{array}$ \\
\hline Vinorelbina & $\begin{array}{l}\text { Muers et al., } \\
2008\end{array}$ & $16 \%$ & $75 \%$ & 6,2 & $9,5(7,5-12,1)$ \\
\hline $\begin{array}{l}\text { Cisplatina + } \\
\text { raltitrexede }\end{array}$ & $\begin{array}{c}\text { van Meerbeeck } \\
\text { et al., } 2005\end{array}$ & $\begin{array}{c}23,6 \% \\
(15,7-31,6)\end{array}$ & $76 \%$ & $5,3(4,6-6,6)$ & $11,4(10,1-15)$ \\
\hline \multirow{2}{*}{$\begin{array}{l}\text { Cisplatina + } \\
\text { gemcitabina }\end{array}$} & $\begin{array}{c}\text { Habib et al., } \\
2013\end{array}$ & $47,6 \%$ & $90,5 \%$ & - & - $^{*}$ \\
\hline & $\begin{array}{c}\text { Kovac et al., } \\
2017\end{array}$ & $50 \%$ & - & 8,6 & 18,6 \\
\hline $\begin{array}{l}\text { Cisplatina + } \\
\text { pemetrexede }\end{array}$ & $\begin{array}{l}\text { Vogelzang et al., } \\
2003\end{array}$ & $\begin{array}{c}41,3 \% \\
(34,8-48,1)\end{array}$ & - & 5,7 & $12,1(10,0-14,4)$ \\
\hline $\begin{array}{l}\text { Cisplatina + } \\
\text { etoposídio }\end{array}$ & $\begin{array}{c}\text { White et al., } \\
2000\end{array}$ & $8 \%$ & $39 \%$ & - & 4,4 \\
\hline $\begin{array}{l}\text { Gemcitabina + } \\
\text { pemetrexede }\end{array}$ & $\begin{array}{l}\text { Millenson et al., } \\
2010\end{array}$ & $0 \%(0-20,6) \ddagger$ & $46 \%$ & $3,3(1,6-5,2)$ & $6,0(3,9-14,0)$ \\
\hline \multirow{2}{*}{$\begin{array}{l}\text { Carboplatina + } \\
\text { pemetrexede }\end{array}$} & $\begin{array}{c}\text { Habib et al., } \\
2013\end{array}$ & $78,9 \%$ & $84,2 \%$ & - & — $^{* *}$ \\
\hline & $\begin{array}{l}\text { Millenson et al., } \\
2010\end{array}$ & $\begin{array}{c}18,8 \% \\
(5,4-41,7) \ddagger\end{array}$ & $94 \%$ & $4,1(1,7-6,6)$ & $13,0(5,6-21,9)$ \\
\hline $\begin{array}{l}\text { Cisplatina + } \\
\text { pemetrexede + } \\
\text { CBP501 }\end{array}$ & Krug et al., 2014 & $31 \%(17,0-47,6)$ & $69 \%(52,4-83)$ & $5,1(3,9-6,5)$ & $13,3(9,2-16,3)$ \\
\hline $\begin{array}{l}\text { Cisplatina }+ \\
\text { pemetrexede }+ \\
\text { axitinib }\end{array}$ & $\begin{array}{l}\text { Buikhuisen et } \\
\text { al., } 2016\end{array}$ & $36 \%$ & $43 \%$ & $5,8(4,6-24)$ & 18,9 \\
\hline $\begin{array}{l}\text { Cisplatina }+ \\
\text { pemetrexede }+ \\
\text { bevacizumabe }\end{array}$ & $\begin{array}{l}\text { Zaleman et al., } \\
2016\end{array}$ & $47 \%$ & $74 \%$ & $9,2(8,5-10,5)$ & $18,8(15,9-22,6)$ \\
\hline $\begin{array}{l}\text { Cisplatina + } \\
\text { pemetrexede + } \\
\text { nintedanib }\end{array}$ & $\begin{array}{l}\text { Grosso et al., } \\
2017\end{array}$ & $56,8 \%$ & - & $9,4(6,7-11,2)$ & $18,3(15,2-28,8)$ \\
\hline $\begin{array}{l}\text { Cisplatina + } \\
\text { pemetrexede + } \\
\text { cediranib }\end{array}$ & Tsao et al., 2018 & $53,0 \%$ & - & 6,9 & 10 \\
\hline $\begin{array}{l}\text { Derivado de } \\
\text { platina + } \\
\text { pemetrexede + } \\
\text { galinpepimut-S }\end{array}$ & $\begin{array}{l}\text { Zauderer et al., } \\
2017\end{array}$ & - & - & $7,4(2,8-14,6)$ & $22,8(9,1-37,6)$ \\
\hline $\begin{array}{l}\text { Cisplatina }+ \\
\text { mitomicina }+ \\
\text { vinblastina }\end{array}$ & $\begin{array}{l}\text { Muers et al., } \\
2008\end{array}$ & $10 \%$ & $72 \%$ & 5,1 & $7,7(6,1-7,9)$ \\
\hline
\end{tabular}

Legendas: IC 95\% - Intervalo de Confiança de 95\%; TRO - Taxa de resposta objetiva, compreende resposta total e parcial à quimioterapia; TCD - Taxa de controle da doença, compreende TRO e aqueles que tiveram a doença estabilizada; TLP - Tempo livre de progressão da doença, em meses; TSG - Tempo de sobrevida global. Notas: - ${ }^{*}$ Não traz mediana de tempo de sobrevida, refere apenas que $41 \%$ dos 21 pacientes submetidos ao regime cisplatina + gemcitabina estavam vivos aos 18 meses de seguimento; — ** Náo traz mediana de tempo de sobrevida, refere apenas que $57,8 \%$ dos 19 pacientes submetidos ao regime carboplatina + pemetrexede estavam vivos aos 18 meses de seguimento; $\ddagger-\mathrm{O}$ intervalo de confiança avaliado no estudo foi de $90 \%$.

e galinpepimut-S apresentaram mediana de tempo livre de progressão superior a seis meses. Já em termos do tempo de sobrevida global, os regimes baseados na associação de cisplatina + gemcitabina, cisplatina + pemetrexede, cisplatina + pemetrexede + axitinib, cisplatina + pemetrexede e bevacizumabe, cisplatina + pemetrexede + nintedanib e derivado de platina + pemetrexede + galinpepimut- $S$ mostraram sobrevidas acima de 16 meses.

Cisplatina associada ao pemetrexede foi o único esquema terapêutico que apresentou diferença média padronizada estatisticamente significante em termos da sobrevida global (Figura 3). 


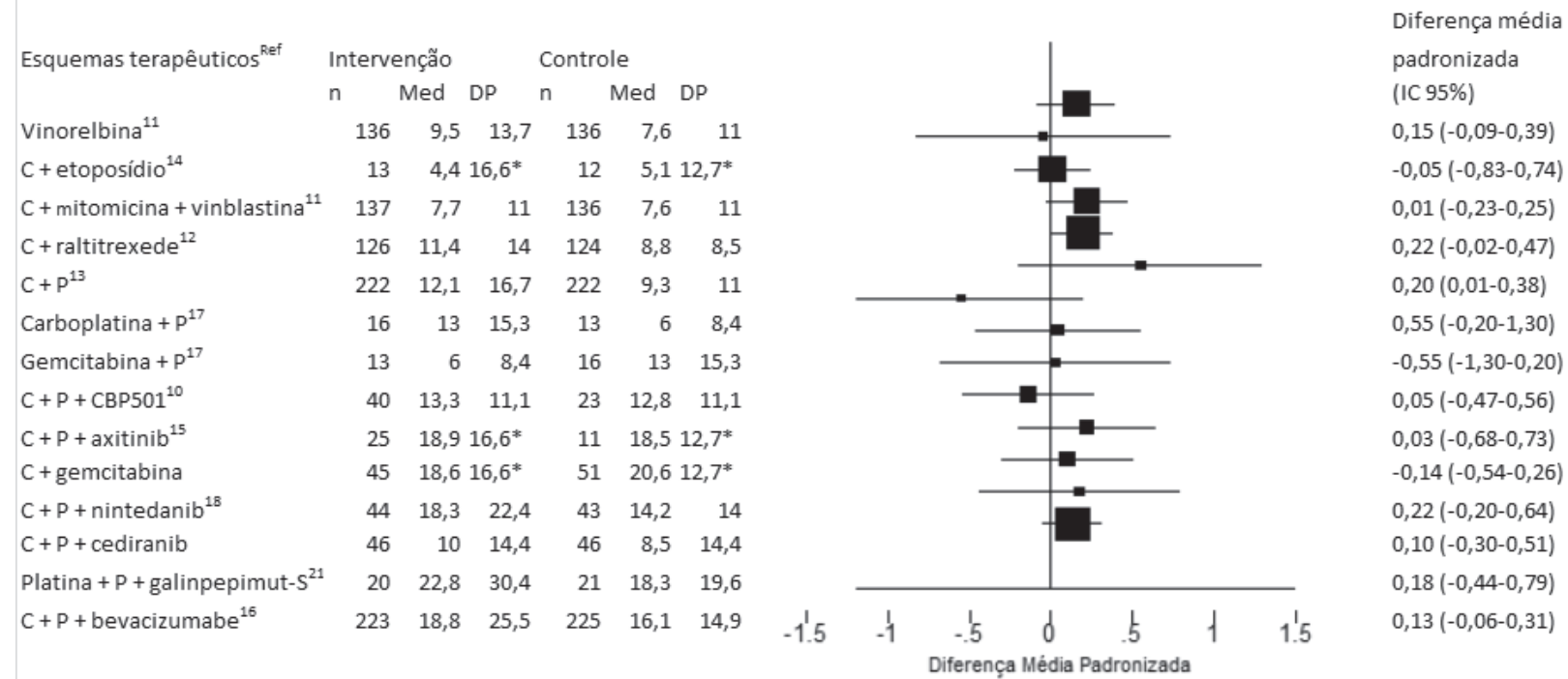

Figura 3. Tempo de sobrevida global (em meses) segundo os esquemas terapêuticos presentes nos estudos incluídos na revisão sistemática

Legendas: Ref. - Número da referência bibliográfica; n - Quantidade de participantes por braço do estudo; Med - Mediana de sobrevida em meses; DP - Desvio-padrão; IC 95\% - Intervalo de confiança de 95\%; C - Cisplatina; P - Pemetrexede.

Nota: * - Valor imputado com a média dos desvios padróes dos outros estudos.

Houve relato de óbito associado a eventos adversos (grau 5) nos estudos de Krug et al. ${ }^{10}$, com esquema quimioterápico associando cisplatina, pemetrexede e CBP501 (2,5\% dos pacientes); Vogelzang et al. ${ }^{13}$ e Grosso et al. ${ }^{19}$, com a associação cisplatina e pemetrexede (1,8\%); Muers et al. ${ }^{11}$, no grupo que utilizou especificamente vinorelbina e cuidados de suporte $(0,9 \%)$; e Zalcman et al. ${ }^{16}$, com a associaçáo cisplatina, pemetrexede e bevacizumabe. Os regimes com maior toxicidade graus 3 e 4 foram gemcitabina associada à cisplatina (leucopenia - 38,1\%; trombocitopenia $23,8 \%$; nausea/vômito - 33\% ${ }^{9}$; cisplatina combinada a pemetrexede (neutropenia - 23,2\% ${ }^{13}$ e $44,6 \%{ }^{16}$ ); cisplatina em combinação com pemetrexede e axitinib (neutropenia $-45 \%)^{15}$, além de cisplatina em combinação com pemetrexede e bevacizumabe (neutropenia - 44,1\% e hipertensão - $23 \%)^{16}$; e cisplatina em combinação com pemetrexede e nintedanib (neutropenia $-43,2 \%)^{19}$.

\section{DISCUSSÃO}

Ainda existe controvérsia sobre o melhor tratamento para o mesotelioma pleural. Uma proporção pequena de pacientes é candidata ao manuseio cirúrgico e, para a maioria dos pacientes, as opçóes disponíveis incluem a quimioterapia, radioterapia ou tratamento de suporte. Entretanto, persistem debates sobre o esquema quimioterápico ideal, com uma variedade de agentes isolados ou em combinaçáo sendo testados e, mais recentemente, sendo exploradas estratégias à base de imunoterapia ${ }^{22}$.
Os esquemas quimioterápicos incluídos nesta revisão foram testados em participantes sem possibilidade de tratamento cirúrgico em quase $90 \%$ dos ensaios randomizados. Dois ensaios clínicos incluíram pacientes que poderiam fazer uma intervenção cirúrgica. Em Buikhuisen et al. ${ }^{15}$, os participantes foram randomizados para dois esquemas quimioterápicos: cisplatina + pemetrexede e cisplatina + pemetrexede + axitinib. O tempo de sobrevida global não diferiu de forma estatisticamente significante nos grupos (18,5 e 18,9 meses, respectivamente) ${ }^{15}$. Já em Zauderer et al. ${ }^{21}$, os participantes também foram randomizados para dois esquemas quimioterápicos: cisplatina + pemetrexede e cisplatina + pemetrexede + galinpepimut-S. O tempo de sobrevida global náo diferiu de forma estatisticamente significante nos grupos por conta do pequeno número de participantes do estudo de fase 2 (18,3 e 22,8 meses, respectivamente $)^{21}$. Galinpepimut-S pode se tornar uma opçáo no tratamento do mesotelioma pleural maligno, caso seja realizado um estudo de fase 3 , com maior número de participantes.

Exceto pelo estudo de Habib et al. ${ }^{9}$, todos os demais examinaram o tempo de sobrevida global. Esse trabalho comparou dois esquemas - cisplatina + gemcitabina $(n=21)$ e carboplatina + pemetrexede $(n=19)$ - e mediu apenas a sobrevida cumulativa em um ano e meio de acompanhamento, correspondente a $41 \%$ e $57 \%$, respectivamente ${ }^{9}$. Na avaliação da qualidade dos artigos, esse estudo foi um dos que apresentou resultado muito negativo. 
O estudo de Kovac et al. ${ }^{18}$ também testou a combinação cisplatina e gemcitabina. Essa combinação foi comparada com cisplatina e pemetrexede. Nos participantes em que a neoplasia progredisse após a quimioterapia, poderia ser utilizado outro tratamento. A combinação cisplatina e gemcitabina teve sobrevida global média inferior de 18,6 meses contra 20,6 da combinação cisplatina e pemetrexede. Os autores sugeriram que cisplatina e gemcitabina podem ser uma opçáo de tratamento de segunda linha do mesotelioma pleural maligno ou, ainda, tratamento de primeira linha nos países onde pemetrexede não está disponível para a população geral ${ }^{18}$.

Nos demais estudos, esquemas baseados em cisplatina e pemetrexede associados a um terceiro antineoplásico apresentaram os maiores tempos de sobrevida global. A associaçáo de bevacizumabe, cisplatina e pemetrexede (18,8 meses, IC 95\% (15,9-22,6), no estudo de Zalcman et al. ${ }^{16}$, mostrou que os participantes possuíam melhor condição clínica e podiam utilizar o pemetrexede como terapia de manutenção ${ }^{16}$. Esse estudo possuiu 448 pacientes, com idade inferior a 76 anos e com menos de $10 \%$ apresentando performance status 2 . Após um tempo de seguimento mediano de 39,4 meses, pacientes que receberam bevacizumabe associado à combinaçáo pemetrexede + cisplatina tiveram uma mediana de sobrevida global melhor do que aqueles que receberam apenas pemetrexede + cisplatina (18,8 vs 16,1 meses, OR ajustada de 0,75 e $\mathrm{p}=0,0167) . \mathrm{Na}$ avaliação da qualidade dos artigos, esse estudo foi um dos que apresentou melhor resultado.

$\mathrm{O}$ estudo de Tsao et al. ${ }^{20}$ utilizou a combinação cisplatina, pemetrexede e cediranib. Este último, um inibidor dos receptores de fator de crescimento endotelial vascular. Após o uso da combinação, os participantes utilizaram cediranib como tratamento de manutenção. Foram 92 participantes, com média de idade de 72 anos. A sobrevida global foi de dez meses contra 8,5 meses da combinaçáo cisplatina, pemetrexede e placebo. A superioridade numérica não obteve, entretanto, significância estatística $(\mathrm{HR}=0,84$ e $\mathrm{p}=0,44)$. $\mathrm{O}$ tempo de sobrevida global ter sido inferior aos outros estudos talvez possa ser explicado pela idade média maior dos participantes ${ }^{20}$.

Outro inibidor dos receptores de fator de crescimento endotelial vascular, nintedanib, foi utilizado em combinação com cisplatina e pemetrexede no estudo de Grosso et al. ${ }^{19}$. O estudo clínico de fase 2, randomizado e duplo-cego, contou com a participaçáo de 87 participantes. Apenas participantes com performance status 0 ou 1 e tumores epitelioide e bifásico na amostra explicam os tempos de sobrevida globais médios altos obtidos. Os participantes que utilizaram o tratamento com nintedanib tiveram tempo de sobrevida global de 18,3 meses contra 14,2 meses do tratamento cisplatina, pemetrexede e placebo. Esse resultado não teve significância estatística $(\mathrm{HR}=0,77$ e $\mathrm{p}=0,319)^{19}$.

O único esquema terapêutico que apresentou diferença média padronizada positiva significante, nessa revisão sistemática, em termos da sobrevida global, foi aquele envolvendo cisplatina associada ao pemetrexede. O estudo de Volgezang et al. ${ }^{13}$ randomizou 448 pacientes para receber cisplatina isolada versus sua associação ao pemetrexede, mostrando melhor sobrevida (12,1 vs 9,3 meses, HR 0,77, $\mathrm{p}=, 02$ ), mas também maior toxicidade (neutropenia, trombocitopenia, vômitos e neutropenia febril) do regime combinado ${ }^{13}$.

A revisão sistemática de Ellis et al. ${ }^{23}$ já tinha identificado o regime pemetrexede + cisplatina como a opção de melhor evidência de eficácia e segurança, com cisplatina + raltitrexede sendo uma alternativa nos casos em que o uso dessa opção não seja possível ${ }^{23}$. Revisão da Cochrane avaliou especificamente a eficácia da combinação pemetrexede + cisplatina, com complementação de ácido fólico e vitamina B12, em pacientes com mesotelioma pleural virgens de tratamento, comparada a outros agentes citotóxicos usados de forma isolada ou combinada e aos cuidados de suporte, e também sinalizou para melhoria da sobrevida em pacientes com bom performance status ${ }^{24}$. Cabe ser destacado que ambas as revisões identificaram apenas o estudo de Volgezang et al. ${ }^{13}$ já mencionado para sustentar esses achados ${ }^{13}$.

A combinação carboplatina + pemetrexede mostrou mediana de tempo de sobrevida global de 13 meses (IC 95\% 5,6-21,9) quando comparada ao esquema gemcitabina + pemetrexede (6 meses, IC 95\% 3,914,0), mas, em um pequeno estudo de fase II com apenas 29 participantes ${ }^{17}$. Esse estudo não pôde ter a sua qualidade avaliada por estar na forma de resumo de congresso. $\mathrm{O}$ estudo teve pacientes menos graves e foi interrompido antes do tempo planejado. Em estudo não randomizado, pertencente a um programa internacional de acesso expandido com 1.704 pacientes, carboplatina + pemetrexede e cisplatina + pemetrexede apresentaram eficácia e segurança comparáveis ${ }^{25}$. A carboplatina parece ser uma opçáo para pacientes que não puderem usar cisplatina e vice-versa.

Em relação à segurança dos esquemas terapêuticos, o uso de antifolatos aumentou a toxicidade da quimioterapia em comparaçáo ao uso isolado de cisplatina. $\mathrm{O}$ estudo de Arnold et al. ${ }^{26}$, com 73 participantes, mostrou que pemetrexede + cisplatina e pemetrexede + carboplatina acarretaram piora no estado de saúde global comparados ao controle ativo de sintomas após 16 semanas $^{26}$. Cisplatina + pemetrexede estiveram associados a três mortes por conta da toxicidade; em Vogelzang et al. ${ }^{13}$, 
todos os casos ocorridos antes de suplementação com ácido fólico e vitamina B12. Desde então, esse uso se tornou corriqueiro na prática clínica sempre que o esquema inclui pemetrexede, com estudos apontando que a adição melhora a eficácia do tratamento, uma vez que a redução dos efeitos adversos permite maior número de ciclos de quimioterapia e maior sobrevida global, comparados aos que não receberam a suplementação ${ }^{27}$.

No Brasil, ainda não há um protocolo clínico ou diretriz terapêutica sobre o tratamento do mesotelioma pleural maligno. Uma série de diretrizes clínicas internacionais recomenda a combinaçáo derivada de platina + antifolato como opção de primeira escolha para o tratamento do mesotelioma pleural maligno nos casos em que apenas a quimioterapia é possível e, também, no tratamento multimodal. Cisplatina e pemetrexede têm a preferência sobre carboplatina e raltitrexede ${ }^{2,23,24,28-30}$. O último guideline da American Society of Clinical Oncology, de 2018, recomenda a associação premetrexede mais platina (qualidade de evidência elevada, com força de recomendaçáo forte) como quimioterapia de primeira linha, com a adiçáo do bevacizumabe podendo ser considerada em pacientes selecionados sem contraindicação ao anticorpo monoclonal (qualidade de evidência elevada, com força de recomendação moderada) ${ }^{31}$. Esses pacientes seriam aqueles com o tipo de mesotelioma epitelial, com performance status 0 - 1 , sem doença cardiovascular significativa, sem hipertensão incontrolada, com idade menor que 75 anos, sem risco de sangramento ou trombos e com valores séricos do fator de crescimento vascular endotelial abaixo da média ${ }^{31,32}$.

Chama atenção o pequeno número de estudos relacionados ao tratamento quimioterápico de mesotelioma pleural identificados nessa revisão. A raridade da condição e os critérios de inclusão aplicados na elegibilidade dos estudos, como a exigência de grupo controle, são possíveis fatores contribuintes para tal. Esse achado reforça a importância de realização de mais ensaios clínicos, sobretudo se considerado o diagnóstico em fase avançada, que limita a possibilidade de tratamento cirúrgico, bem como a expectativa de que o número de casos se mantenha significativo ainda por muitos anos no país, visto que a proibição da extração, industrialização, comercialização e distribuição do amianto no país pelo Supremo Tribunal Federal só ocorreu em novembro de $2017^{33}$.

Apenas em dez estudos foi possível realizar a avaliação da qualidade ${ }^{9-16,19,21}$. Os outros compreenderam três resumos apresentados em congressos ${ }^{17,18,20}$. A falta do mascaramento adequado e a ausência do relato de como foi feito o processo de randomizaçáo sugerem a possibilidade da presença de vieses nos estudos. Embora a inclusão de resumos de congressos seja recomendada para evitar viés de publicação, deve-se ter cautela no uso dessas referências ${ }^{34}$.

O presente estudo apresenta algumas limitaçóes. É difícil comparar os resultados dos desfechos entre estudos que apresentam diferenças no tempo médio de acompanhamento, idade, histologia, performance status, entre outros fatores. Por se tratar de esquemas terapêuticos distintos, não foi possível verificar quais desses fatores objetivamente afetaram o prognóstico da doença. Sendo assim, há risco de vieses se tentarmos comparar os resultados dos desfechos apresentados entre os estudos.

\section{CONCLUSÃO}

Ensaios clínicos futuros em mesotelioma pleural maligno devem utilizar a dupla derivada de platina + antifolato como grupo controle. A combinaçáo de cisplatina e pemetrexede tem a preferência sobre carboplatina e raltitrexede por ter sido mais testada. A adição de bevacizumabe à terapia derivada de platina + antifolato, embora passe a ser opção terapêutica para casos menos graves no guideline da American Society of Clinical Oncology, precisa de mais estudos.

Em uma doença com prognóstico tão ruim como o mesotelioma, qualquer novo tratamento prometendo paliação dos sintomas ou aumento da sobrevida pode parecer muito atraente. $\mathrm{Na}$ avaliação de novas terapias, no entanto, é importante considerar qual a melhor opçáo para tais pacientes, considerando além da eficácia o perfil de segurança. Pacientes e provedores de cuidado devem pesar a maior eficácia do uso de derivados de platina combinado aos antifolatos com a toxicidade apresentada por esses esquemas. Faz-se importante também o desenvolvimento de estudos clínicos com desenhos metodológicos adequados. Por fim, em relação à eventual incorporação de tecnologias, há a necessidade de realização de avaliaçóes econômicas que possam servir de embasamento para uma decisão sobre a melhor opção para o tratamento quimioterápico do mesotelioma pleural maligno em cada país.

\section{CONTRIBUIÇÕES}

André de Oliveira Souza participou da concepção, busca nas bases de dados, seleção de artigos, extração de dados, avaliação da qualidade dos artigos e redação final. Vera Lúcia Edais Pepe participou da concepção, busca nas bases de dados, avaliação da qualidade dos artigos e redação final. Lenice Gnocchi da Costa Reis participou da concepção, avaliação da qualidade dos artigos e redação final. Rosângela Caetano participou da concepção e redação final. Luiz Gustavo André Oliveira participou da 
seleçáo de artigos e extração de dados. Todos os autores aprovaram a versão final do artigo.

\section{DECLARAÇÃO DE CONFLITO DE INTERESSES}

Nada a declarar.

\section{FONTES DE FINANCIAMENTO}

Não há.

\section{REFERÊNCIAS}

1. Terra Filho M, Freitas JBP, Nery LE. Doenças asbesto-relacionadas. J Bras Pneumol. 2006;32(2 Suppl):S48-53. doi: http://dx.doi.org/10.1590/S180637132006000800009.

2. Pinto C, Novello $S$, Torri $V$, et al. Second Italian consensus conference on malignant pleural mesothelioma: state of the art and recommendations. Cancer Treat Rev. 2013;39(4):328-339. doi: https://doi.org/10.1016/j. ctrv.2012.11.004.

3. Bianchi C, Bianchi T. Malignant Mesothelioma: Global incidence and relationship with asbestos. Ind Health. 2007;45(3):379-387. doi: https://doi.org/10.2486/ indhealth.45.379.

4. Iwatsubo Y, Pairon JC, Boutin C, et al. Pleural mesothelioma: dose-response relation at low levels of asbestos exposure in a French population-based case-control study. Am J Epidemiol. 1998;148(2):133142. doi: https://doi.org/10.1093/oxfordjournals.aje. a009616.

5. Kazan-Allen L. Asbestos and mesothelioma: worldwide trends. Lung Cancer. 2005;49(1 Suppl):S3-8. doi: https://doi.org/10.1016/j.lungcan.2005.03.002.

6. Algranti E, Saito CA, Carneiro APS, et al. The next mesothelioma wave: mortality trends and forecast to 2030 in Brazil. Cancer Epidemiol. 2015;39(5):687-692. doi: https://doi.org/10.1016/j.canep.2015.08.007.

7. Zucali PA, De Vincenzo F, Simonelli M, et al. Future developments in the management of malignant pleural mesothelioma. Expert Rev Anticancer Ther. 2009;9(4):453-467. doi: https://doi.org/10.1586/ era.09.2.

8. Higgins JPT, Green S, editors. Cochrane handbook for systematic reviews of interventions Version 5.1.0. London: Cochrane. [2006?]- [updated 2011Mar 20; cited 2018 Aug 4. Available from: https://training. cochrane.org/handbook.

9. Habib EE, Fahmy ES. Chemotherapy management of malignant pleural mesothelioma: a phase II study comparing two popular chemotherapy regimens. Clin Transl Oncol. 2013;15(11):965-968. doi: https://doi. org/10.1007/s12094-013-1015-3
10. Krug LM, Wozniak AJ, Kindler HL, et al. Randomized phase II trial of pemetrexed/cisplatin with or without CBP501 in patients with advanced malignant pleural mesothelioma. Lung Cancer. 2014;85(3):429-434. doi: https://doi.org/10.1016/j.lungcan.2014.06.008.

11. Muers MF, Stephens RJ, Fisher P, et al. Active symptom control with or without chemotherapy in the treatment of patients with malignant pleural mesothelioma (MS01): a multicentre randomised trial. Lancet. 2008 May17;371(9625):1685-1694. doi: https://doi. org/10.1016/S0140-6736(08)60727-8.

12. van Meerbeeck JP, Gaafar R, Manegold C, et al. Randomized phase III study of cisplatin with or without raltitrexed in patients with malignant pleural mesothelioma: an intergroup study of the European Organisation for Research and Treatment of Cancer Lung Cancer Group and The National Cancer Institute of Canada. J Clin Oncol. 2005;23(28):6881-6889. doi: https://doi.org/10.1200/JCO.20005.14.589.

13. Vogelzang NJ, Rusthoven JJ, Symanowski J, et al. Phase III study of pemetrexed in combination with cisplatin versus cisplatin alone in patients with malignant pleural mesothelioma. J Clin Oncol. 2003;21(14): 2636-2644. doi: https://doi.org/10.1200/JCO.2003.11.136.

14. White SC, Anderson H, Jayson GC, et al. Randomised phase II study of cisplatin-etoposide versus infusional carboplatin in advanced non-small-cell lung cancer and mesothelioma. Ann Oncol. 2000;11(2):201-206. doi: https://doi.org/10.1023/A:1008328605413.

15. Buikhuisen WA, Scharpfenecker M, Griffioen AW, et al. A randomized phase II study adding axitinib to pemetrexed-cisplatin in patients with malignant pleural mesothelioma: a single-center trial combining clinical and translational outcomes. J Thorac Oncol. 2016;11(5):758-768. doi: https://doi.org/10.1016/j. jtho.2016.01.014.

16. Zaleman G, Mazieres J, Margery J, et al. Bevacizumab for newly diagnosed pleural mesothelioma in the Mesothelioma Avastin Cisplatin Pemetrexed Study (MAPS): a randomised, controlled, open-label, phase 3 trial. Lancet 2016;387(10026):1405-1414. doi: https:// doi.org/10.1016/S0140-6736(15)01238-6.

17. Millenson MM, Lee J, Hanna NH, et al. Pemetrexed (Pem) plus gemcitabine (Gem) or carboplatin (Carbo) in patients (pts) with advanced malignant mesothelioma (MPM): A randomized phase II trial, E1B03. J Clinical Oncol. 2010;28(15 Suppl):e18053. doi: https://doi. org/10.1200/jco.2010.28.15_suppl.e18053.

18. Kovac V, Zwitter M, Strbac D, et al. Cisplatin with pemetrexed or gemcitabine in prolonged infusion for inoperable mesothelioma: a phase ii randomized trial. J Thorac Oncol. 2017;12(1 Suppl):S1362-S1363. doi: https://doi.org/10.1016/j.jtho.2016.11.1929. 
19. Grosso F, Steele N, Novello S, et al. Nintedanib plus pemetrexed/cisplatin in patients with malignant pleural mesothelioma: phase II results from the randomized, placebo-controlled LUME-Meso Trial. J Clin Oncol. 2017;35(31):3591-3600. doi: https://doi.org/10.1200/ JCO.2017.72.9012.

20. Tsao AS, Miao J, Wistuba II, et al. SWOG S0905: a randomized phase II study of cediranib versus placebo in combination with cisplatin and pemetrexed in chemonaive patients with malignant pleural mesothelioma. J Clin Oncol. 2018;36(15 Suppl):8514-8514. doi: https://doi. org/10.1200 / JCO.2018.36.15_suppl.8514.

21. Zauderer MG, Tsao AS, Dao T, et al. A randomized phase II trial of adjuvant galinpepimut-s, WT-1 analogue peptide vaccine, after multimodality therapy for patients with malignant pleural mesothelioma. Clin Cancer Res. 2017;23(24): 7483-7489. doi: https://doi. org/10.1158/1078-0432.CCR-17-2169.

22. Cinausero M, Rihawi K, Sperandi F, et al. Chemotherapy treatment in malignant pleural mesothelioma: a difficult history. J Thorac Dis. 2018 Jan;10(Suppl 2):S304-310. doi: https://doi.org/10.21037/jtd.2017.10.19.

23. Ellis P, Davies AM, Evans WK, et al. The use of chemotherapy in patients with advanced malignant pleural mesothelioma: a systematic review and practice guideline. J Thorac Oncol. 2006;1(6):591-601. doi: https://doi.org/10.1016/S1556-0864(15)30366-X.

24. Green JA, Dundar Y, Dodd SR, et al. Pemetrexed disodium in combination with cisplatin versus other cytotoxic agents or supportive care for the treatment of malignant pleural mesothelioma. Cochrane Database Syst Rev. 2007 Jan;(1): CD005574. doi: https://doi. org/10.1002/14651858.CD005574.pub2.

25. Santoro A, O'Brien ME, Stahel RA, et al. Pemetrexed plus cisplatin or pemetrexed plus carboplatin for chemonaïve patients with malignant pleural mesothelioma: results of the International Expanded Access Program. J Thorac Oncol. 2008;3(7):756-763. doi: https://doi. org/10.1097/JTO.0b013e31817c73d6.

26. Arnold DT, Hooper CE, Morley A, et al. The effect of chemotherapy on health-related quality of life in mesothelioma: results from the SWAMP trial. Br J Cancer. 2015;112(7):1183-1189. doi: https://doi. org/10.1038/bjc.2015.77.

27. Scagliotti GV, Shin DM, Kindler HL, et al. Phase II study of pemetrexed with and without folic acid and vitamin B12 as front-line therapy in malignant pleural mesothelioma. J Clin Oncol. 2003;21(8):1556-1561. doi: https://doi.org/10.1200/JCO.2003.06.122.

28. van Zandwijk N, Clarke C, Henderson D, et al. Guidelines for the diagnosis and treatment of malignant pleural mesothelioma. J Thorac Dis. 2013;5(6):E254-E307. doi: https://doi.org/10.3978/j.issn.2072-1439.2013.11.28.
29. Scherpereel A, Astoul P, Baas P, et al. Guidelines of the European Respiratory Society and the European Society of Thoracic Surgeons for the management of malignant pleural mesothelioma. Eur Respir J. 2010;35(3):479495. doi: https://doi.org/10.1183/09031936.00063109.

30. Baas P, Fennell D, Kerr KM, et al. Malignant pleural mesothelioma: ESMO Clinical Practice Guidelines for diagnosis, treatment and follow-up. Ann Oncol. 2015 Sep;26(5 Suppl):v31-v39. doi: https://doi.org/10.1093/ annonc/mdv199.

31. Kindler HL, Ismaila N, Armato SG, et al. Treatment of malignant pleural mesothelioma: american society of clinical oncology clinical practice guideline. J Clin Oncol. 2018 May;36(13):1343-1373. doi: https://doi. org/10.1200/JCO.2017.76.6394.

32. Kondola S, Manners D, Nowak AK. Malignant pleural mesothelioma: an update on diagnosis and treatment options. Ther Adv Respir Dis. 2016;10(3) 275-88. doi: https://doi.org/10.1177/1753465816628800.

33. Dundar Y, Dodd S, Williamson P, et al. Case study of the comparison of data from conference abstracts and fulltext articles in health technology assessment of rapidly evolving technologies: does it make a difference? Int J Technol Assess Health Care. 2006;22(3):288-294. doi: https://doi.org/10.1017/S0266462306051166.

Recebido em 13/11/2018 Aprovado em 30/4/2019 